\title{
Film forming behavior in thin film lubrication at high speeds
}

\author{
He LIANG, Dan GUO*, Jianbin LUO* \\ State Key Laboratory of Tribology, Tsinghua University, Beijing 100086, China \\ Received: 23 December 2016 / Revised: 16 February 2017 / Accepted: 24 March 2017 \\ (C) The author(s) 2017. This article is published with open access at Springerlink.com
}

\begin{abstract}
The film forming condition may transit into thin film lubrication (TFL) at high speeds when it is under severe starvation. Central film thicknesses and film thickness profiles are obtained via a technique of relative optical interference intensity. These profiles show a critical film thickness lower than which the absolute values of the film thickness gradient against speed or time decrease. It is possible to be in the thin film lubrication mode under such conditions. The high speed flow drives the lubricant molecules to rearrange in TFL and critical film thickness higher than $100 \mathrm{~nm}$ is achieved. The viscosity is one of the main factors controlling the decreasing rate and the critical film thickness. This paper is designed to investigate the thin film lubrication behavior at high speeds.
\end{abstract}

Keywords: thin film lubrication; thin EHL film; high speeds; starvation

\section{Introduction}

In a nanoscale the lubrication behavior may be determined by both physical characteristics of the lubricant and the interaction between lubricant molecules and solid surfaces. It is defined as thin film lubrication (TFL) [1-4], which is a transition between elastohydrodynamic lubrication (EHL) [5-7] and boundary lubrication (BL) [8, 9]. In TFL ordered layers are formed essentially by aligned molecules driven by shearing or flowing in a confined space and these layers determine the behavior of the lubrication as has been proven both theoretically and experimentally $[1-4,10,11]$. It results in higher effective viscosity as well as thicker film thickness in TFL compared with that of absorbed layers in BL as long as the shear rate is less than the critical value of approximately $10^{6}-10^{8} \mathrm{~s}^{-1}$ for shear thinning in confined lubricants [12]. Furthermore the side leakage and fluidity are minimized compared with EHL. Some factors like an external electrical field that have the ability to change the orientation of molecules can also change the film thickness and friction characteristics in TFL [13].

Most of the researches on thin film lubrication have been conducted under very low speed, for instance $10 \mathrm{~mm} / \mathrm{s}$, in order to reach the necessary nanoscale. It is hypothesized that there can be thin film lubrication at high speeds as long as the two friction surfaces are close enough to form a nano gap of tens of nanometers. Such condition is normally one of severely starved lubrication. The published researches on starvation mainly focus on the declining flow and the starvation degree [14-16]. The relationship between the inlet replenishment and the central film thickness was studied $[17,18]$. The inlet replenishment is mainly driven by surface tension as well as van del Waals force which might be important for an extremely thin film $[19,20]$. The asymmetric film thickness distribution induced by the centrifugal effects must be considered for high speeds $[16,21]$. The lubrication condition and the characteristics in TFL at high speeds still need to be investigated as the film thickness falls down to tens of nanometers.

In the current work, the study of thin film lubrication is extended to high speeds and the basic properties for thin film lubrication are proposed for these highspeed conditions. The influence of factors like the viscosity, speed, and oil supply is investigated.

* Corresponding authors: Dan GUO, E-mail: guodan26@tsinghua.edu.cn; Jianbin LUO, E-mail: luojb@tsinghua.edu.cn 


\begin{tabular}{|c|c|c|c|}
\hline \multicolumn{4}{|c|}{ Nomenclature } \\
\hline EHL & Elastohydrodynamic lubrication & $n$ & The interference order \\
\hline TFL & Thin film lubrication & \multirow[t]{2}{*}{$I, I_{\max }, I_{\min }$} & \multirow{2}{*}{$\begin{array}{l}\text { The light intensity, the maximum and } \\
\text { minimum interference light intensity }\end{array}$} \\
\hline BL & Boundary Lubrication & & \\
\hline$t_{0}$ & Operating temperature $(\mathrm{K})$ & \multirow[t]{2}{*}{$\bar{I}$} & \multirow{4}{*}{$\begin{array}{l}\text { The relative interference light intensity } \\
\left(\bar{I}=\left(2 I-I_{\max }-I_{\min }\right) /\left(I_{\max }-I_{\min }\right)\right) \\
\text { The relative light intensity of zero film } \\
\text { thickness }\end{array}$} \\
\hline$\eta_{0}$ & Ambient viscosity $(\mathrm{Pa} \cdot \mathrm{s})$ & & \\
\hline$u$ & Lubricant entrainment speed, $u=\left(u_{1}+u_{2}\right) / 2$ & \multirow[t]{2}{*}{$\bar{I}_{0}$} & \\
\hline$u_{1}, u_{2}$ & Surface velocities of disc and ball (m/s) & & \\
\hline$E^{\prime}$ & Reduced modulus $(\mathrm{Pa})$ & & \multirow{6}{*}{$\begin{array}{l}\text { The revolution of disc } \\
\text { The time for operating the test } \\
\text { The radius of the oil track on the disc } \\
\text { The decreasing gradient of film thickness } \\
\text { against entrainment speed defined in } \\
\text { Eq. (2) }\end{array}$} \\
\hline$p_{\mathrm{H}}$ & Maximum Herzian pressure (Pa) & $t$ & \\
\hline$b$ & Theoretical Hertz contact radius (m) & $r$ & \\
\hline$h$ & $\begin{array}{l}\text { Lubricant film thickness (m) } \\
\text { Central film thickness (m) }\end{array}$ & $\psi, \psi_{1}, \psi_{2}$ & \\
\hline $\begin{array}{l}h_{\mathrm{c}} \\
h_{\mathrm{cff}}\end{array}$ & $\begin{array}{l}\text { Central film thickness (m) } \\
\text { Central film thickness under fully flooded }\end{array}$ & & \\
\hline & lubrication $(\mathrm{m})$ & & \\
\hline$\lambda$ & The wavelength of the incident light & \multirow[t]{2}{*}{$\lambda_{\mathrm{t}}$} & \multirow{2}{*}{$\begin{array}{l}\text { The decreasing gradient of film thickness } \\
\text { against time defined in Eq. (4) }\end{array}$} \\
\hline & The reflective index of lubricant & & \\
\hline
\end{tabular}

\section{Experimental condition}

All the tests were conducted at up to $30 \mathrm{~m} / \mathrm{s}$ on a custom made high-speed ball-on-disc test rig whose properties and functions were first introduced in Ref. [16]. A highly polished ball was loaded normally on a disc coated with a chromium layer. The roughnesses of the ball and the disc were $5 \mathrm{~nm}$ and $3 \mathrm{~nm}$, respectively. Compared to the tens of nanometers of the film thickness formed between the ball and the disc, the influence of the roughness could be ignored. The ball and the disc were driven by high-speed spindles and the rotating speeds were controlled individually. The oil was introduced through near the contact inlet employing an oil-jet system. All tests were carried out at pure rolling and at a constant load of $15 \mathrm{~N}$ such that the maximum Hertz pressure in the contact zone was approximately $431 \mathrm{MPa}$. The room temperature was a constant $25^{\circ} \mathrm{C}$. Table 1 shows the material properties of the balls and discs used in the tests. The coated glass disc gives optical access to the contact so that the relative optical interference intensity (ROII) technique $[2,13]$ can be utilized to measure the film thickness. The following equation [22] was used to determine the film thickness in the contact zone:

$h=\frac{\lambda}{4 \pi k}\left[\left(n+\left|\sin \frac{n \pi}{2}\right|\right) \cdot \pi+\arccos (\bar{I}) \cdot \cos n \pi-\arccos \left(\overline{I_{0}}\right)\right]$

where $\lambda, k$, and $n$ represent the wavelength of the incident light, reflective index of lubricant and interference order respectively. $I, I_{\max }$ and $I_{\min }$ are the light intensity, and the maximum and minimum interference light intensity, respectively, $\bar{I}$ is used as the relative interference light intensity $\left(\bar{I}=\left(2 I-I_{\max }-I_{\min }\right) /\right.$ $\left.\left(I_{\max }-I_{\min }\right)\right)$ and $\bar{I}_{0}$ represents the relative light intensity of zero film thickness.

Table 1 Operating conditions for measurements.

\begin{tabular}{|c|c|c|c|c|c|}
\hline \multirow{2}{*}{$\begin{array}{l}\text { Operating } \\
\text { conditions }\end{array}$} & Load $(\mathrm{N})$ & $E^{\prime}(\mathrm{GPa})$ & $p_{\mathrm{H}}(\mathrm{MPa})$ & $b(\mu \mathrm{m})$ & $t_{0}\left({ }^{\circ} \mathrm{C}\right)$ \\
\hline & 15 & 117 & 431 & 128 & 25 \\
\hline \multirow{3}{*}{$\begin{array}{c}\text { Material } \\
\text { properties }\end{array}$} & & Material & Radius (mm) & Roughness (nm) & \\
\hline & Ball & Steel & 11.1125 & 5 & \\
\hline & Disc & Glass with $\mathrm{Cr}$ coating & 45 & 3 & \\
\hline
\end{tabular}


Three viscosities of polyalphaolefin (PAO) oil were used in the test and their physical properties measured by a viscometer (Anton Paar, Austria) are shown in Table 2.

Table 2 Lubricant properties $\left(t_{0}=25^{\circ} \mathrm{C}\right)$.

\begin{tabular}{cccc}
\hline & PAO2 & PAO4 & PAO8 \\
\hline$\eta_{0}(\mathrm{mPa} \cdot \mathrm{s})$ & 6.96 & 29.59 & 86.55 \\
$\rho\left(\mathrm{kg} / \mathrm{m}^{3}\right)$ & 798.8 & 819.0 & 832.6 \\
\hline
\end{tabular}

\section{Results}

\subsection{The film forming under severe starvation at high speeds}

Figure 1 shows the measured central film thickness under fully flooded lubrication and under starved lubrication at speeds up to $30 \mathrm{~m} / \mathrm{s}$ on the dual logarithmic coordinate. Three kinds of oil with various viscosities were tested (shown in Table 2).
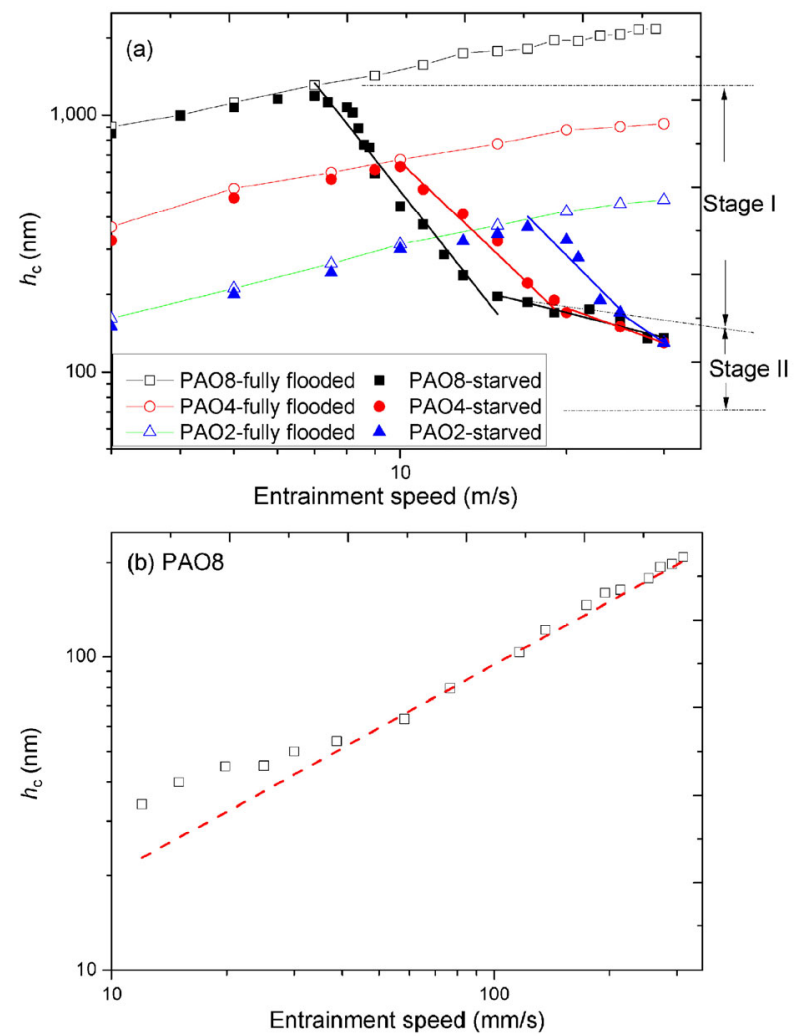

Fig. 1 Measurements of fully flooded and starved central film thickness for a range of speeds, pure rolling, $p_{\mathrm{H}}=431 \mathrm{MPa}, t_{0}=$ $25^{\circ} \mathrm{C}$, oil supply: $4.25 \mathrm{~mL} / \mathrm{s}$ for fully flooded lubrication and $0.85 \mathrm{~mL} / \mathrm{s}$ for starved lubrication.
For fully flooded lubrication, the central film thicknesses increase with speed and can achieve hundreds of nanometers. It is under elastohydrodynamic lubrication (EHL) as the typical horseshoe-shaped film thickness distribution can be obtained from the interference images shown in Ref. [16]. At very low speed, the central film thickness deviates from the traditional Hamrock-Dowson curve, which shows transition from EHL to TFL. The critical film thickness is approximately $50 \mathrm{~nm}$ at $30 \mathrm{~mm} / \mathrm{s}$ for PAO8.

For starved lubrication, the central film thicknesses starts to decrease exponentially with speed beyond a critical speed and can be divided into two stages (shown in Fig. 1(a)). The second stage occurs when the film thickness reaches a low value beyond which the film thickness decreases more slowly against the entrainment speed. In order to compare the decreasing rate for the two stages, the decreasing gradient of central film thickness against entrainment speed is defined as

$$
\psi=\operatorname{abs}\left[\log \left(\frac{h_{1}}{h_{2}}\right) / \log \left(\frac{u_{1}}{u_{2}}\right)\right]
$$

The parameter $\psi$ at two stages (named as $\psi_{1}$ and $\psi_{2}$ ) is found using curve fitting. A higher value of $\psi$ represents a quicker decreasing rate. The shifting of the parameter $\psi$ suggests that the lubrication condition might transition from EHL into thin film lubrication.

Figure 2 compares the trend of critical central film thickness and the parameter $\psi$ as function of viscosity.

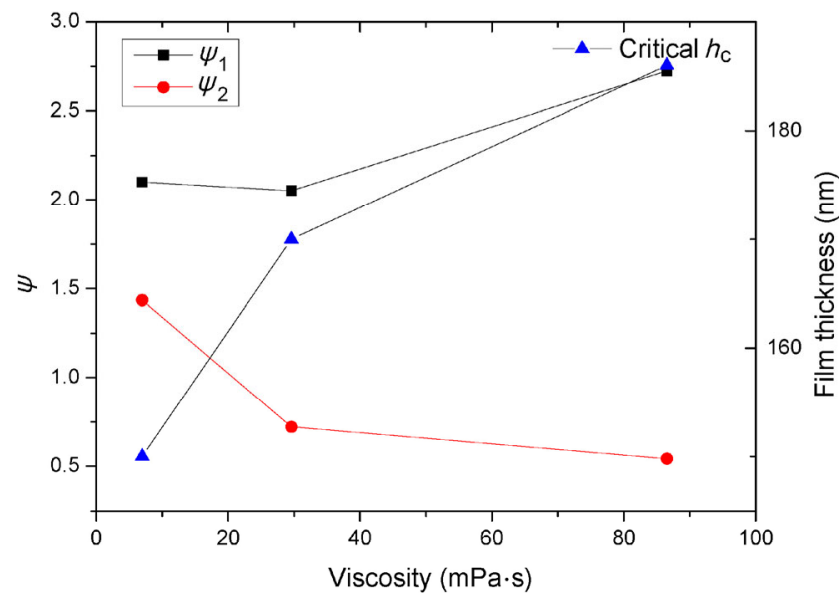

Fig. 2 Change of critical film thickness and the paramter $\psi$ against viscosity for starved lubrication, pure rolling, $p_{\mathrm{H}}=431 \mathrm{MPa}, t_{0}=$ $25{ }^{\circ} \mathrm{C}$, oil supply: $0.85 \mathrm{~mL} / \mathrm{s}$. 
The critical film thickness shows a slight lowering, from $190 \mathrm{~nm}$ to $150 \mathrm{~nm}$ with decreasing viscosity. For one specific oil, the value of $\psi_{2}$ is smaller than that of $\psi_{1}$, which shows that the decreasing rate of film thickness against entrainment speed is slowed. Using PAO8 as an example, it can be seen from Fig. 2 that the value of $\psi_{2}$ is 0.54 , one-fifth of the value of $\psi_{1}$. Furthermore, the changes of $\psi_{1}$ and $\psi_{2}$ show the opposite trend against increasing viscosity. The two stages might be influenced by different mechanisms. In stage I, the higher viscosity oil achieves a higher absolute value of $\psi_{1}$. The main factor should be the centrifugal effect [21]. The centrifugal force is a bulk force mainly determined by the mass and the rotating speed. The oil with higher viscosity gets a higher mass since the film thickness increases at a certain speed. This leads to a stronger centrifugal effect. The centrifugal force has the ability to weaken the oil replenishment and to accelerate the reduction of film thickness under starved lubrication as it can drive off the oil near the inlet zone and accelerate the side leakage. Therefore in stage I the change of film thickness is mainly determined by the lubricant bulk properties. On the contrary in stage II, the higher viscosity oil gets a lower value of $\psi_{2}$. This might be controlled by both the lubricant properties and the interfacial force between the lubricant molecules and the solid surface as the centrifugal effects are weakened for such thin film thickness.

Mid-plane film thickness profiles both parallel to and perpendicular to the entrainment speed direction are shown in Fig. 3. For the film thickness profiles parallel to the entrainment speed direction, the film shows to be flat in the center and the minimum film thickness appears near the outlet zone of the contact. These are important characteristics for EHL lubrication. In stage I of starvation, the film thickness decreases but still maintains the character of EHL, although the deviation of minimum film thickness and the central film thickness is getting smaller. In stage II of starvation, the reduction of film thickness near the outlet zone disappears. The whole profile shows to be flat. For the film thickness profiles perpendicular to the entrainment speed direction, the film thickness is convex in the center, and the film thickness at the edge of the contact zone decreases. As the film thickness is reduced, the film thickness profiles become flatter. It shows that
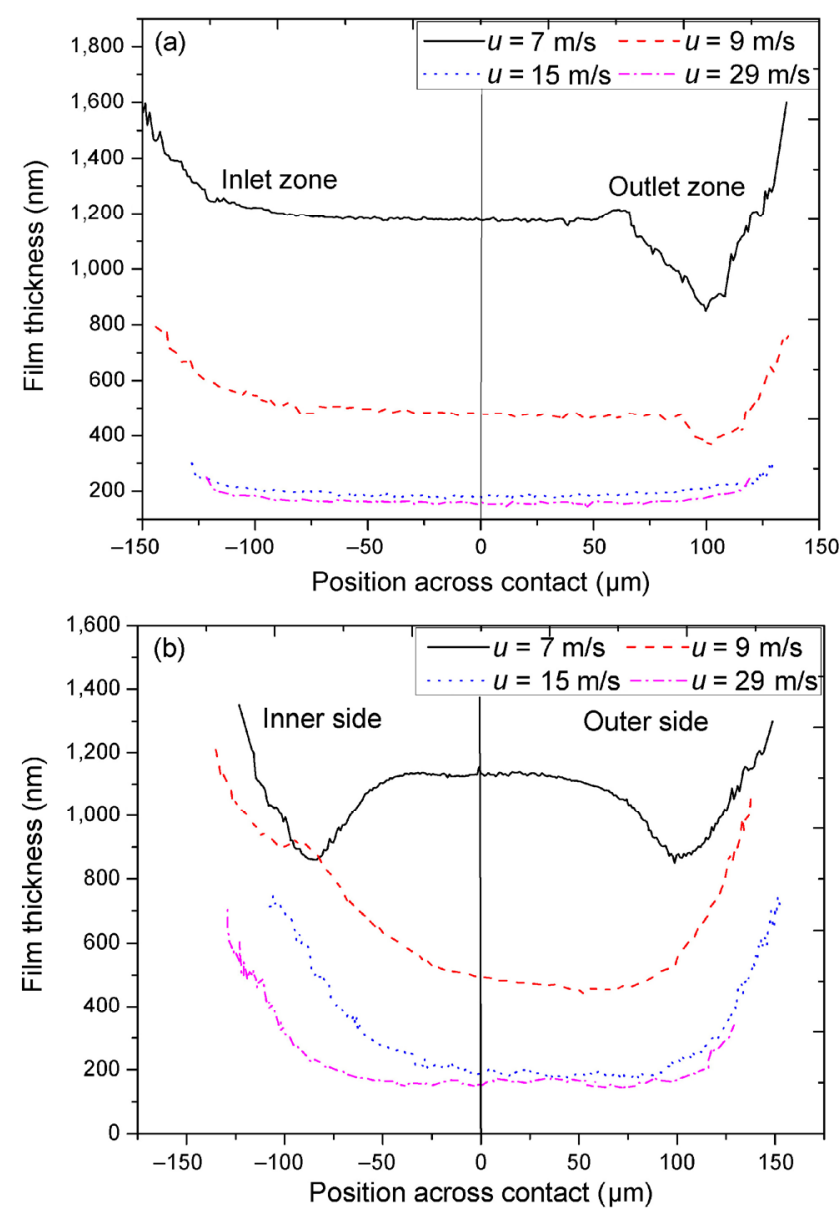

Fig. 3 Mid-plane film thickness profiles (a) parallel to and (b) perpendicular to the direction of entrainment speed under starved lubrication. The perpendicular axis is along the direction of centrifugal forces. Pure rolling, $p_{\mathrm{H}}=431 \mathrm{MPa}, t_{0}=25^{\circ} \mathrm{C}$, oil supply: $51 \mathrm{~mL} / \mathrm{min}$, PAO8.

the side leakage is weakened to an extent and the flow law may not fit this kind of condition.

At high speeds and under starved lubrication conditions the critical film thickness of approximately $170 \mathrm{~nm}$ is much higher than the typical transition film thickness of less than $100 \mathrm{~nm}$ at lower speeds, although it still shows the characteristics of thin film lubrication. Figure 4 shows the measured film thickness of various oil supplies under starved lubrication for PAO8. It can be seen that the starvation starts at lower entrainment speed for a lower oil supply. Furthermore, it shows two stages in the starvation region as the deviation between $\psi_{1}$ and $\psi_{2}$ still exists as shown in Fig. 5. The reduction of oil supply makes the critical film thickness of stage II slowly decrease. However, it does not change the opposite trend of $\psi_{1}$ and $\psi_{2}$ 


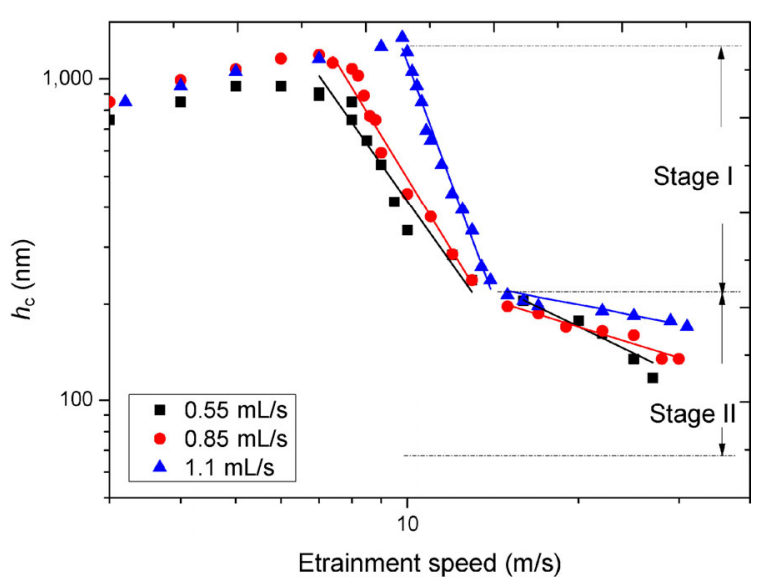

Fig. 4 Measurements of starved film thickness for three different oil supply rate, pure rolling, $p_{\mathrm{H}}=431 \mathrm{MPa}, t_{0}=25^{\circ} \mathrm{C}, \mathrm{PAO} 8$.

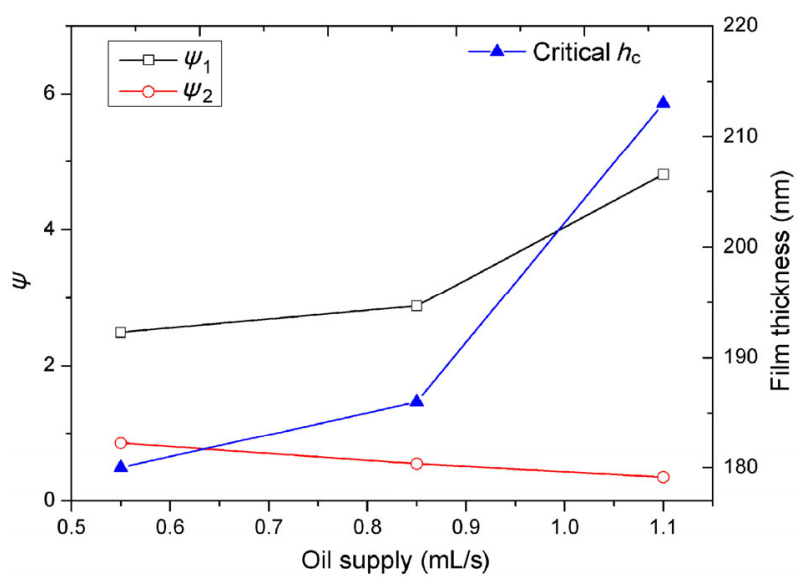

Fig. 5 Changes of the paramter $\psi$ in stage I $\left(\psi_{1}\right)$ and stage II $\left(\psi_{2}\right)$ against oil supply rate, pure rolling, $p_{\mathrm{H}}=431 \mathrm{MPa}, t_{0}=25{ }^{\circ} \mathrm{C}$, PAO8.

against entrainment speed, as the value of $\psi_{1}$ increases with oil supply while the value of $\psi_{2}$ decreases with oil supply. It shows that the properties in stage II, which are supposed to be in TFL, cannot be determined by oil supply.

\subsection{The film forming behavior for limited oil supply at high speeds}

In the above tests, the oil was supplied continuously for both fully flooded lubrication and starved lubrication. In this section, supply of oil was controlled and it was observed that film thickness gradually decreased against time from fully flooded lubrication to severe starvation after the oil supply was suspended as shown in Fig. 6 for oil of various viscosities at $30 \mathrm{~m} / \mathrm{s}$.
The film thickness is expected to decline all the time following the reduction of inlet replenishment. The fitting curves are drawn based on the following equation, first proposed by Chevalier et al. [18] and modified by Liang et al. [21] considering the replenishment and the centrifugal effects.

$$
\begin{aligned}
& R\left(n_{\mathrm{d}}\right)=\frac{1}{\sqrt[\gamma]{c_{\mathrm{n}} n_{\mathrm{d}}+1 / r_{0}^{\gamma}}} \\
& R(t)=\frac{1}{\sqrt[\gamma]{c_{\mathrm{t}} t+1 / r_{0}^{\gamma}}}
\end{aligned}
$$

where $R=h_{\mathrm{c}} / h_{\text {cff }}, c_{n}=(2 \pi r / u) c_{\mathrm{t}} . n_{\mathrm{d}}$ and $t$ stand for the revolution of the disc and operating time. Parameter $c_{\mathrm{t}}$ represents the asymptotic film thickness decay rate and parameter $\gamma$ physically means the side flow.

It appears that at a film thickness of approximately $100 \mathrm{~nm}$, the declining rate of film thickness against time slows down. Therefore, the film thickness is subdivided into two stages as shown in Fig. 6. In stage I, the reduction of film thickness can be described by Eq. (3); while in stage II, the film thickness deviates from the fitting curves proposed by Eq. (3) and decreases exponentially with time. A uniform parameter $\lambda_{\mathrm{t}}$ is defined to describe this decreasing gradient of film thickness against time in stage II.

$$
\lambda_{\mathrm{t}}=\operatorname{abs}\left[\log \left(\frac{h_{1}}{h_{2}}\right) / \log \left(\frac{t_{1}}{t_{2}}\right)\right]
$$

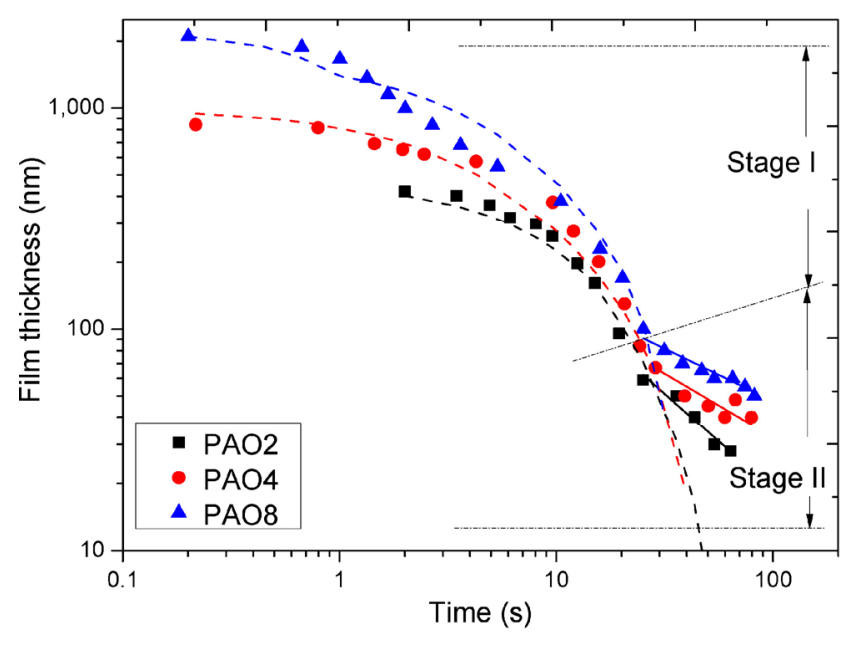

Fig. 6 Measurements of starved central film thickness against time after the oil supply is suspended for three different oil, pure rolling, $p_{\mathrm{H}}=431 \mathrm{MPa}, t_{0}=25^{\circ} \mathrm{C}$. 
It is noted that the oil with higher viscosity gets a higher value of critical film thickness and a smaller $\lambda_{\mathrm{t}}$ as shown in Fig. 7. The critical film thickness in this condition is less than $100 \mathrm{~nm}$. The parameter $\lambda_{\mathrm{t}}$ increases with increasing viscosity, which is a trend similar to that of the parameter $\psi_{2}$ in the last section.

Figure 8 shows the film thickness declining with time after the oil is stopped for PAO2 with different speeds from $10 \mathrm{~m} / \mathrm{s}$ to $30 \mathrm{~m} / \mathrm{s}$. The experimental results indicate that the film thicknesses diverge and get thicker from the general fluid theory when a critical film thickness is reached.

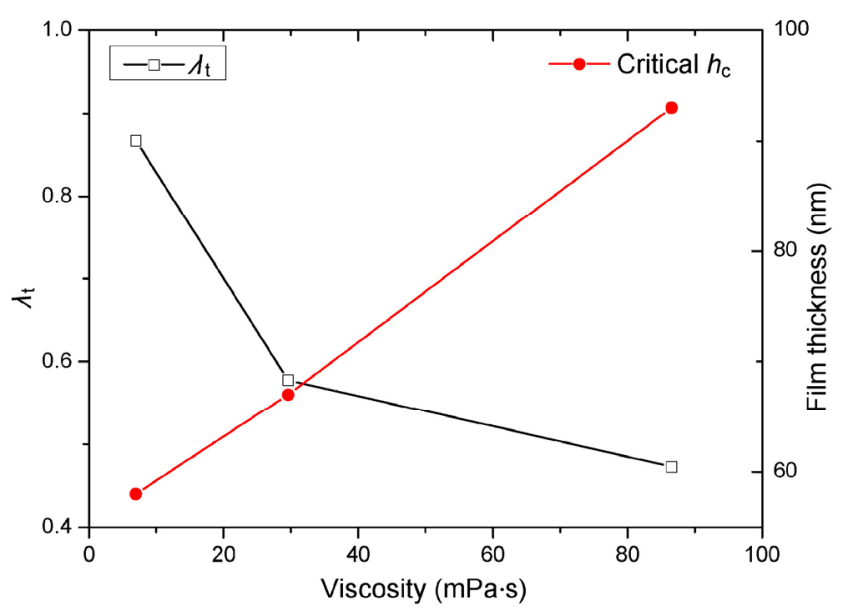

Fig. 7 Changes of critical filmthickness and the parameter $\lambda_{\mathrm{t}}$ against oil viscosity, pure rolling, $p_{\mathrm{H}}=431 \mathrm{MPa}, t_{0}=25^{\circ} \mathrm{C}, \mathrm{PAO}$.

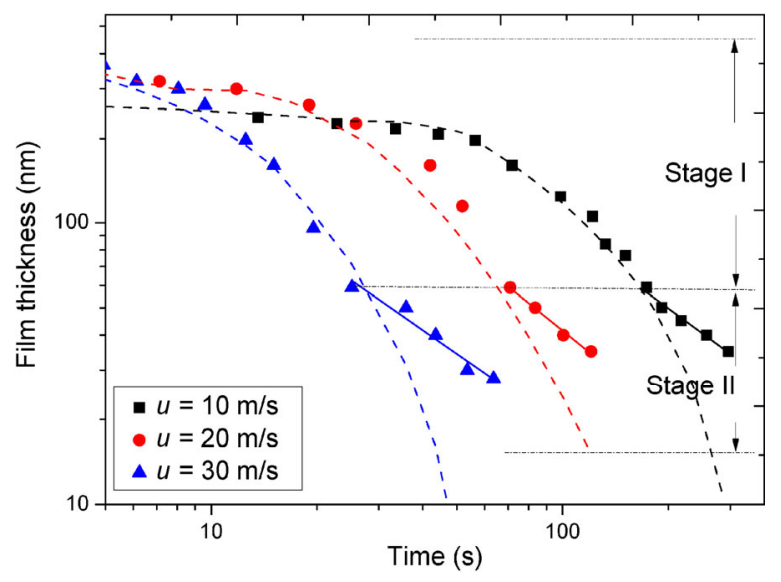

Fig. 8 Measurements of starved central film thickness against time after the oil supply is suspended for a range of speeds, pure rolling, $p_{\mathrm{H}}=431 \mathrm{MPa}, t_{0}=25^{\circ} \mathrm{C}, \mathrm{PAO} 2$.

\section{Discussion}

As a conclusion of the above tests, it is shown that the film thickness in starved lubrication can always be subdivided into two stages. In stage I, the film thickness decreases with entrainment speed or time following the starvation theory. The film thickness can be controlled by the hydrodynamic effects and the bulk properties of the oil. In stage II, the film thickness deviates from the classical theory, it decreases slower with entrainment speed or time. Both of these results indicate that the lubrication transits from EHL to thin film lubrication condition.

As a transition region between EHL and BL, both the dynamic film and the ordered lubricant film exist in the thin film lubrication condition. The ratio of ordered film layer to the whole film thickness rises largely enough as the film thickness decreases to nanoscale so that the influence of the ordered film layer can no longer be ignored.

It can be explained by the increasing effective viscosity mainly due to the formation of ordered molecule layers. The aligned layers are caused by both of the lubricant properties and the interfacial forces between the lubricant molecules and the solid surface.

There are two main forces affecting the lubricant molecules. One is the driven force, parallel to the entrainment speed. The shearing effects or high speed flow rate can drive the molecule to move in the same direction of flow. The other is vertical to the entrainment speed. It is the interfacial force between the lubricant molecules and solid surfaces that aligns the molecules to the solid surface. Under such confined actions, the lubricant molecules near the solid surface can be organized as solid-like which achieve a higher effective viscosity than the bulk viscosity.

The characteristics for thin film lubrication at high speeds are shown as follows.

It is possible that the high speed flow rate is the main driving force for aligning the molecules at high speeds. In EHL theory, the dynamic flow governs the characteristics of lubrication and film forming, so the adhesive film and the aligned molecular layer are relatively thin and can be ignored. In thin film lubrication, both the ordered layer and the dynamic 
flow layer exist. The ordered degree of the ordered layer decreases gradually from the surface to the fluid film. The influence of the ordered layer is strong enough to change the properties of film forming. The critical film thickness for PAO8 oil at lower speeds under fully flooded lubrication is about $50 \mathrm{~nm}$. The experimental results at pure rolling show that the high speed flowing rate might have the ability to drive molecules to arrange the direction and the ordered layer can influence the lubrication characteristics at a higher film thickness as the critical film thickness of TFL at high speeds is increased, for instance, $58 \mathrm{~nm}$ for $\mathrm{PAO} 2$ and $93 \mathrm{~nm}$ for PAO8 with a limited oil supply.

The critical film thickness and decreasing gradient are influenced by viscosity, which differs from that under EHL. According to general fluid theory, high viscosity may help prevent oil from replenishing, and then the film thickness decreasing gradient against speed or time would be enhanced. Besides, the high viscosity oil will undergo high centrifugal effects under the same speed, which also intensify the film thickness reduction under starved lubrication. However, in thin film lubrication, it shows the opposite trend. Oil with higher viscosity gets higher effective viscosity, decreases with speed or time more slowly on the contrary and keeps thicker film thickness. The flat film thickness profile suggests that the side leakage is weakened.

\section{Conclusion}

In this study, the measurement of decreasing film thickness under constant oil supply or constant speed is discussed. The results show the characteristics of thin film lubrication under severe starvation at high speeds when the film thickness is less than a critical film thickness. Driven by high speed flow, ordered layers are formed, which determine the lubrication behavior in thin film lubrication by increasing the effective viscosity. The study shows that in these conditions the critical film thickness is lower than that under lower speeds and in fully flooded condition. The viscosity influences the decreasing gradient of film thickness against entrainment speed and time.

\section{Acknowledgments}

The work is financially supported by National Natural Science Foundation of China (Nos. 51375255, 51321092, and 51527901).

Open Access: The articles published in this journal are distributed under the terms of the Creative Commons Attribution 4.0 International License (http:// creativecommons.org/licenses/by/4.0/), which permits unrestricted use, distribution, and reproduction in any medium, provided you give appropriate credit to the original author(s) and the source, provide a link to the Creative Commons license, and indicate if changes were made.

\section{References}

[1] Luo J, Wen S. Mechanism and characters of thin film lubrication at nanometer scale. Science in China, Series AMathematics, Physics, Astronomy 39(12): 1312-1322 (1996)

[2] Luo J, Wen S, Huang P. Thin film lubrication. Part I. Study on the transition between EHL and thin film lubrication using a relative optical interference intensity technique. Wear 194(1): 107-115 (1996)

[3] Luo J, Liu S. The investigation of contact ratio in mixed lubrication. Tribol Int 39(5): 409-416 (2006)

[4] Luo J, Huang P, Wen S, Li L K. Characteristics of liquid lubricant films at the nano-scale. J Tribol 121(4): 872-878 (1999)

[5] Lugt P M, Morales-Espejel G E. A review of elastohydrodynamic lubrication theory. Tribol Trans 54(3): 470496 (2011)

[6] Spikes H A. Sixty years of EHL. Lubr Sci 18(4): 265-291 (2006)

[7] Zhu D, Wang Q J. Elastohydrodynamic Lubrication: A gateway to interfacial mechanics-Review and prospect. J Tribol 133(4): 041001 (2011)

[8] Hu Y, Ma T, Wang H. Energy dissipation in atomic-scale friction. Friction 1(1): 24-40 (2013)

[9] Zhang J, Meng Y. Boundary lubrication by adsorption film. Friction 3(2): 115-147 (2015)

[10] $\mathrm{Hu}$ Y Z, Granick S. Microscopic study of thin film lubrication and its contributions to macroscopic tribology. Tribol Lett 5(1): 81-88 (1998)

[11] Zhang S, Liu Y, Luo J. In situ observation of the molecular ordering in the lubricating point contact area. J Appl Phys 116(1): 014302 (2014) 
[12] Itoh S, Ishii K, Fukuzawa K, Zhang H. Shear Thinning of nanometer-thick liquid lubricant films measured at high shear rates. Tribol Lett 53(3): 555-567 (2014)

[13] Luo J, Shen M, Wen S. Tribological properties of nanoliquid film under an external electric field. J Appl Phys 96(11): 6733-6738 (2004)

[14] Cann P M E, Damiens B, Lubrecht A A. The transition between fully flooded and starved regimes in EHL. Tribol Int 37(10): 859-864 (2004)

[15] Liu J, Wen S. Fully flooded, starved and parched lubrication at a point contact system. Wear 159(1): 135-140 (1992)

[16] Liang H, Guo D, Luo J. Experimental investigation of lubrication film starvation of polyalphaolefin oil at high speeds. Tribol Lett 56(3): 491-500 (2014)

[17] Damiens B, Venner C H, Cann P M E, Lubrecht A A. Starved lubrication of elliptical EHD contacts. J Tribol 126(1):

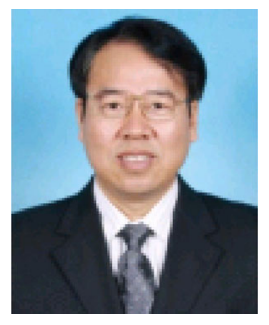

Jianbin LUO. He received his BEng degree from Northeastern University in 1982, and got his MEng degree from Xi'an University of Architecture and Technology in 1988. In 1994, he received his $\mathrm{PhD}$ degree from Tsinghua University and then joined the faculty of Tsinghua University. Prof. Jianbin Luo is an academician of the Chinese Academy of Sciences and a Yangtze River Scholar Distinguished

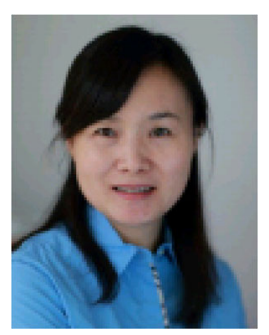

Dan GUO. She received the M.S. degree in engineering mechanics in 1995 from Xi' an Jiaotong University and Ph.D. degree in engineering mechanics in 1999 from Tsinghua University. She joined the State Key Laboratory of Tribology at

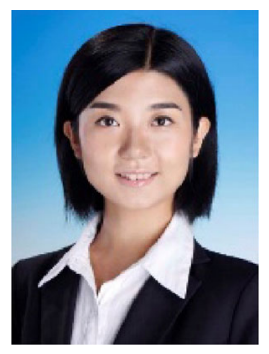

He LIANG. She received her Ph.D. degree in 2015 from State Key Lab of Tribology, Tsinghua University, China. The same year she joined the
105-111 (2004)

[18] Chevalier F, Cann P, Colin F, Dalmaz G, Lubrecht A. Film thickness in starved EHL point contacts. J Tribol 120(1): 126-133 (1998)

[19] Guangteng G, Spikes H. The role of surface tension and disjoining pressure in starved and parched lubrication. Proc Inst Mech Eng Part J: J Eng Tribol 210(2): 113-124 (1996)

[20] Jacod B, Pubilier F, Cann P, Lubrecht A. An analysis of track replenishment mechanisms in the starved regime. Tribology Series 36: 483-492 (1999)

[21] Liang H, Guo D, Ma L, Luo J. Experimental investigation of centrifugal effects on lubricant replenishment in the starved regime at high speeds. Tribol Lett 59(1): 1-9 (2015)

[22] Ma L, Zhang C. Discussion on the technique of relative optical interference intensity for the measurement of lubricant film thickness. Tribol Lett 36(3): 239-245 (2009)

Professor of Tsinghua University, Beijing, China. He was awarded the STLE International Award (2013), the Chinese National Technology Progress Prize (2008), the Chinese National Natural Science Prize (2001), and the Chinese National Invention Prize (1996). Prof. Luo has been engaged in the research of thin film lubrication and tribology in nanomanufacturing. He was invited as a keynote or plenary speaker for 20 times on the international conferences.

Tsinghua University from 1999. Her current position is a professor and the deputy director of the laboratory. Her research areas cover the mechanism of interaction among nanoparticles, lubrication and failure mechanism under rigorous conditions, and movement behavior of confined-microfluidic.

Tribology Group at Imperial College London as a postdoc. Her research areas involve the mechanism of lubrication behaviour in lubricated contacts and in rolling element bearings at high speeds. 